\title{
Graduation exams for medical students: A proposal.
}

Shaza Hamad

shamad@horus.edu.eg

\begin{abstract}
Scoring the clinical performance for graduating medical and dental students are multifactorial. Given the outbreak of COVID-19, the resultant global health crisis requires applying unprecedent measures to evaluate these students in a safe, yet effective, environment. This proposal suggests two scenarios for designing the medical graduation exams by the end of May 2020 and introduces a dynamic conceptual model for evaluating the suggested proposal.
\end{abstract}

\section{Keywords}

Graduation exams, Medicine and Dentistry, Testing and evaluation, Pedagogy, Virtual testing portal. 


\section{Introduction}

The global health crisis we are living threatens lives and poses collateral damage to the economic, social, and educational levels. Nevertheless, the post-outbreak consequences would pose dire socio-economic and health threats if unqualified doctors were approved of as graduates. Therefore, care and extra-ordinary effort, along with effective planning, must be exerted to avoid such problems. To that end, decision making and governmental policies must be planned so that smart solutions this digital world may accept are put into immediate action.

Within the domain of teaching and evaluating science, technology, engineering, and mathematics, Martin et al. (2019) concluded that a deficit-based perspective evoked many interventions of which effectiveness could not be promised. Therefore, no specific intervention sufficed to be implemented globally, leaving much space for innovative intervention to manage unprecedented crises. Given the tremendous challenges the COVID-19 pandemic has imposed on testing final-year medical students, proposals are suggested to guarantee the validity and reliability of high-stakes performance assessments whilst protecting the safety of all participants. The Objective Structured Examination of Practical Skills (OSEPS) were evaluated by 'hybrid' stations, and 15 x 12-minute stations, including history taking, clinical examination, and explanation/advice skills being tested, together with clinical decision making, diagnostic acumen, and management planning. Special measures in a pandemic environment can be taken to conduct clinical examinations safely. The general administrative procedures on each of the three days of the examination included isolated participant registration spots at entry after measuring the temperature of each applicant, investigating the travel history, applying rigorous measures of infection control, and sanitization. Zoom cloud was used for exam briefing, and social distancing was kept at a one-meter distance among each participant. the open buffets were canceled. Student-Patient contact was kept to the critical minimum, with specialized nurses assigned to check the applied measures strictly (Boursicot et al. 2020). The following sections 
describe a two-scenario proposal to prepare efficient graduation exams for medical and dental students in governmental and private Egyptian universities.

\section{Proposal}

Various universities applied Just-In-Time Teaching after the outbreak of COVID-19 as an active learning pedagogy that includes knowledge-based questions in multiple-choice format, integrated case studies, and student responses prior to the actual lecture session (Madiraju et al. 2020). However, testing the outcome of e-learning is not explored. Campus exams included, routinely, written, clinical and oral exams. The first scenario is much hoped for in case of managing the outbreak by the end of May 2020: Optimistic Scenario. In this scenario, students can sit their exams in extremely clean and disinfected campus settings where final-year students are only examined. This would include conventional written-exams and hypothetical clinical exams (without real cases). The clinical exam can be based on an oral discussion of the usual procedures the final-year students would take to manage several virtual cases. This scenario should not impede the scoring and grading for all students.

If matters complicated, the safety of patients and the would-be fresh graduates must be prioritized. To find off-campus alternatives that can evaluate the candidates require simulation of the campus environment at an electronic platform that can be accessed all over the country. The challenges are place, cases, invigilation, the effectiveness of evaluation, and preparation of students for the changing nature of exams.

To solve the problem of place, invigilation, and clinical examination of cases, all distinguished professors, from all specialties, can integrate effort with IT specialists and graphic designers to create a software program that simulates the clinic and clinical practice. This application can be programmed to generate as many questions as the enduser, usually an average professor, would like to have after being uploaded on a cloud. The cloud would be accessible by national ID numbers and customized passwords. The place of sitting the exams can be all ICDL centers and Computer-based examination centers. 
These centers are equipped with firewalls that prevent access to other websites. As a physical place, students can enter these spots without any studying material.

For written exams, all questions must be phrased as multiple-choice questions or "true" and "false" (as two options) to avoid the limitation of string algorithms to the case-sensitive answers. As long as the student selects an answer, the online e-grading system works perfectly. One of the possible options is also asking several questions about uploaded images. Providing the system with 100 questions and enabling the shuffling option will generate several versions of the exam, which will not be easily distributed or even predicted by students, especially if the time limit will not exceed 1 or 2 hours. Essay-form and shortanswer questions are not recommended. If the exam is comprehensive enough to cover the syllabus and the time limit is predetermined, the criteria of evaluation shall be met. Compiling a bank of 10,000 questions from which all examiners can generate their customized versions of exams on a secured and supervised platform would boost the success of this experiment.

For oral exams, online sessions can be scheduled on Microsoft Teams, Google Meet, Webex, or Zoom Cloud to which students can join using their national IDs. These online sessions can be recorded and posted to the university for further assessment. These records would serve as electronic answer sheets.

Examiners shall only provide a list of questions with model answers. Online meetings can include 3 to 6 students who will be invited by their IDs. Examiners can either be professors, assistant lecturers or teaching assistants, given that the questions and the model answers are already designed by distinguished professors. Moreover, the meeting will be recorded so that professors can double-check the assessment process if they have doubts. With a list of 50 questions, one examiner, and six students in each 30-minute session, five examiners can examine 300 students in 10 sessions (5 hours only). Given that all departments can have this number of staff members, this should simulate the real environment of oral 
exams. Assigning lecturers from other nearest specialty might be indicated for small-sized academic paramedical departments (e.g., pathologists can help histologists).

Clinical exams, the most challenging ones, can be interactive testing facilitated by the university with a new website that is hosting a 3D simulation of the patient. It should simulate the process of clinical examination that asks students about the required procedure in each stage (disinfecting, medical history, charting, diagnosing, ...etc.). Students will be directed to the next question based on their answers to each question until they fill out the chart. All questions can be generated from archival cases that have been already diagnosed based on clinical pictures, radiographs, histopathology, and lab investigations. Other customized options can be made. This includes distributing dummy heads on which students can work on and upload a video for their work to be assessed.

Spending a 3-month internship in a teaching hospital should be mandated as a prerequisite for graduation after passing the online exams. The conceptual model of the proposed intervention is shown in Figure 1. 


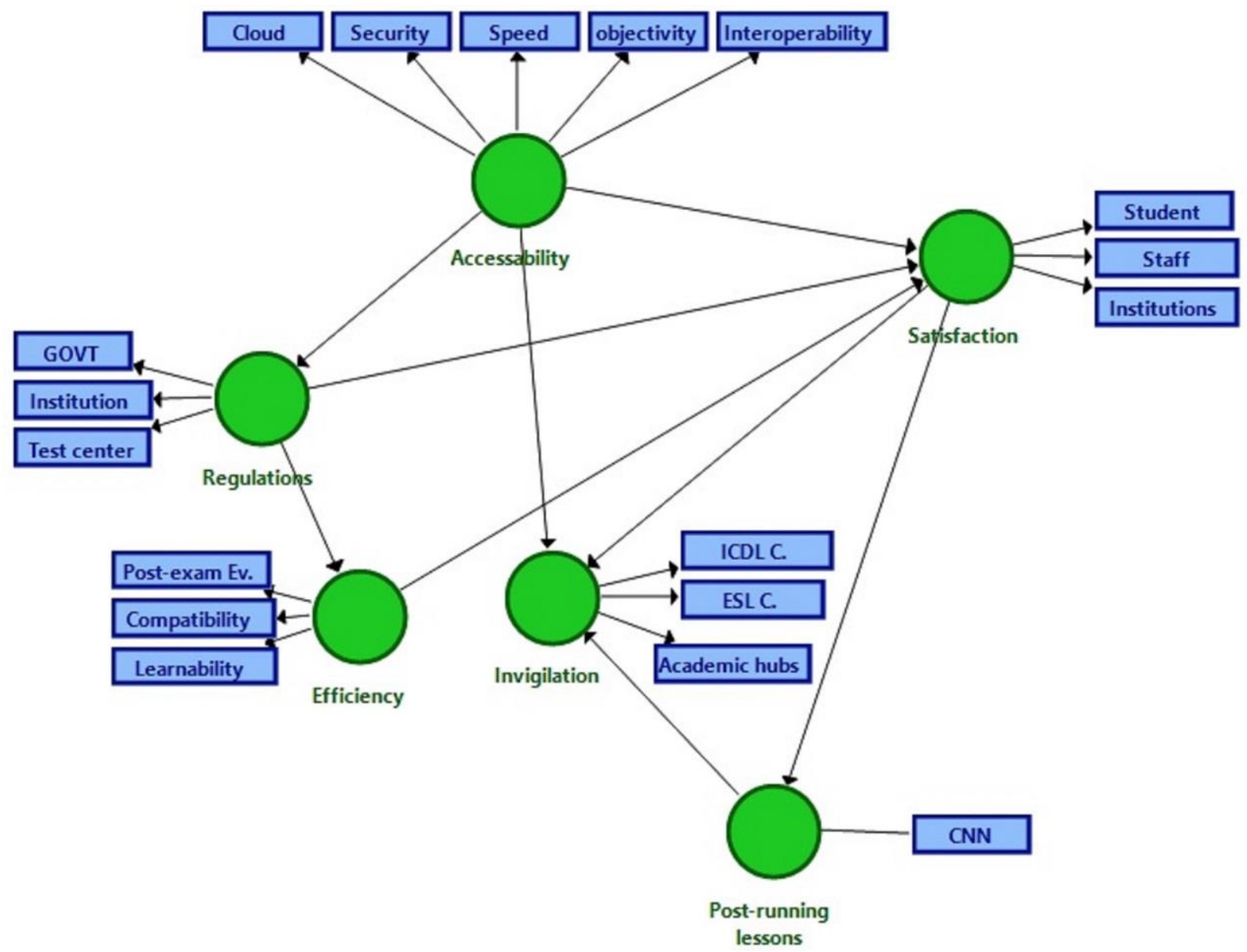

Figure 1. Proposed conceptual model

The main variables of the models are efficiency, invigilation, regulations, accessibility, post-running lessons, and satisfaction. The latent variables are defined in Figure 1.

When the testing portal is ready, teaching assistants can take all the tests to report any problems. Their results can be scored. The moderating variables can be calculated using structural equation modeling and analytical hierarchical processing model. Several proposed models used exploratory structural equation modeling to predict the success rate of building up a virtual examination environment (Pedram et al. 2020; Reubenson et al., 2020).

There is no perfect option that fits all private and governmental universities equally. However, less-equipped universities can hire testing portals from equipped universities that 
will succeed in constructing it. For the individual home-based poor internet connection, ICDL centers can be used to host all students. Shuffling questions and using clinical data can reduce cheating attempts to the critical minimum. Building up a giant portal from which all average professors can generate their exams easily would not add threats of rejections to this experiment. Satisfaction are expected to be $70 \%$ on the first run.

\section{Conclusion}

Although this global health crisis poses substantial challenges on evaluating final-year medical and dental students, the use of technology to face this challenge is more problematic to the least developed countries. All institutional efforts, national and international, should be integrated to contribute to managing this global priority. Interdepartmental and intra-departmental integration is also required to help all recipient, be they decision-makers or medical students, visualize the idea. 


\section{References}

Boursicot, K., Kemp, S., Ong, T. H., Wijaya, L., Goh, S. H., Freeman, K., \& Curran, I. (2020). Conducting a high-stakes OSCE in a COVID-19 environment. MedEdPublish, 9.

Madiraju, C., Tellez-Corrales, E., Hua, H., Stec, J., Nauli, A. M., \& Brown, D. M. (2020). Analysis of Student Perceptions of Just-In-Time Teaching Pedagogy in PharmD Microbiology and Immunology Courses. Frontiers in immunology, 11, 351.

Martin, J. P., Choe, N. H., Halter, J., Foster, M., Froyd, J., Borrego, M., \& Winterer, E. R. (2019). Interventions supporting baccalaureate achievement of Latinx STEM students matriculating at 2-year institutions: A systematic review. Journal of Research in Science Teaching, 56(4), 440-464.

Reubenson, A., Ng, L., \& Gucciardi, D. F. (2020). The Assessment of Physiotherapy Practice tool provides informative assessments of clinical and professional dimensions of student performance in undergraduate placements: a longitudinal validity and reliability study. Journal of Physiotherapy.

Pedram, S., Palmisano, S., Skarbez, R., Perez, P., \& Farrelly, M. (2020). Investigating the process of mine rescuers' safety training with immersive virtual reality: A structural equation modelling approach. Computers \& Education, 103891. 